\title{
La délectation du rasa. La tradition esthétique de l'Inde
}

Delighting in rasa. An aesthetic tradition of India.

\section{Philippe Bruguière}

\section{(2) OpenEdition}

Journals

Édition électronique

URL : http://journals.openedition.org/ethnomusicologie/1298

ISSN : 2235-7688

Éditeur

ADEM - Ateliers d'ethnomusicologie

Édition imprimée

Date de publication : 31 décembre 1994

Pagination : $3-26$

ISBN : 2-8257-0503-9

ISSN : 1662-372X

\section{Référence électronique}

Philippe Bruguière, «La délectation du rasa. La tradition esthétique de l'Inde », Cahiers

d'ethnomusicologie [En ligne], 7 | 1994, mis en ligne le 03 janvier 2012, consulté le 19 avril 2019. URL :

http://journals.openedition.org/ethnomusicologie/1298

Ce document a été généré automatiquement le 19 avril 2019.

Tous droits réservés 


\title{
La délectation du rasa. La tradition esthétique de l'Inde
}

\author{
Delighting in rasa. An aesthetic tradition of India.
}

\section{Philippe Bruguière}

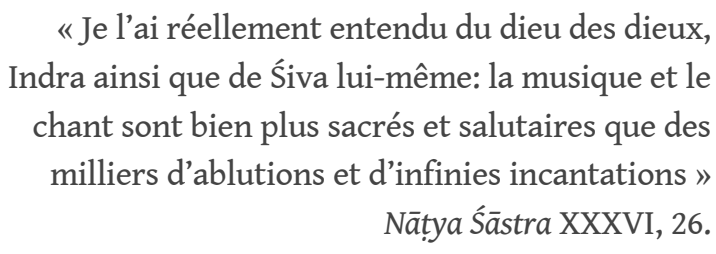

Bien avant que la réflexion sur l'art ne devienne dans l'Europe des Lumières une matière que l'on désigna sous le nom d'esthétique ${ }^{1}$, les philosophes de l'Antiquité en avaient implicitement formulé les grandes idées qui, de la renaissance au romantisme, alimentèrent théories et créations artistiques. Cette nouvelle discipline, tantôt regardée comme la science du Beau, tantôt comme celle du Sublime ou celle des jugements de goût, fut l'objet de nombreuses spéculations (dont certaines encore vivaces aujourd'hui) qui s'efforcèrent d'en donner une définition en multipliant les variations sur le thème du Beau. Peu satisfaisantes d'un point de vue méthodologique et épistémologique, ces théories du Beau ou du jugement de goût furent vivement critiquées à la fin du siècle dernier et c'est ainsi que fut proposée une nouvelle perspective, acceptée de la plupart des esthéticiens contemporains, celle qui consistait à envisager l'esthétique comme la science générale ou universelle de l'art ${ }^{2}$.

2 L'art fut dès lors regardé comme un fait positif dont l'étude des lois qui régissent l'ensemble des œuvres présente autant d'intérêt que celles que l'on peut observer dans la nature ou celles qui structurent le monde des êtres vivants. En redéfinissant les fondements de son passé philosophique pour diriger dès le début du XX $\mathrm{XX}^{\mathrm{e}}$ siècle une partie de son analyse méthodologique vers la sociologie, la psychologie ou la linguistique, l'esthétique occidentale est aujourd'hui devenue une discipline intégrant de multiples points de vue, systématisant par l'unité de son objet la variété des méthodes qui peuvent s'y appliquer (Souriau 1970:132). 
3 L'histoire de cette forme de la pensée réflexive sur l'art connut en Inde un tout autre cheminement. Alors que l'on retrouve dans les systèmes philosophiques des penseurs hellénistes certaines des idées fondamentales qui caractérisent la théorie esthétique de l'Inde, celle-ci fut très explicitement exposée dès l'apparition du premier traité d'art dramatique, le Nātya Śāstra ${ }^{3}$, rédigé au tout début de l'ère chrétienne et attribué au sage Bharata ${ }^{4}$. Ce texte, véritable rhétorique de l'art qui aurait été révélé à Bharata par le dieu Brahmā $\bar{a}^{5}$, est toujours regardé aujourd'hui par la tradition artistique comme la première autorité en matière d'esthétique et demeure une réference incontournable en matière de dramaturgie et de critique littéraire. Le mot nātya qui, dans son acceptation courante, signifie danse et représentation mimée, englobe ici toutes les disciplines qui participent à l'art théâtral, telles la poésie, la musique, la scénographie, l'architecture ou la peinture. Ces différentes branches artistiques, qui possèdent chacune leurs propres modalités de représentation, sont envisagées dans le rapport spécifique qu'elles entretiennent avec le théâtre. La théorie de Bharata les rassemble toutes dans une même pensée à la fois unifiante et réductionniste qui passe sous silence l'analyse individuelle de chacune d'entre elles. Seuls seront par la suite minutieusement expliqués à la lumière de cette théorie, les procédés et mécanismes littéraires qu'il convient de mettre en œuvre pour que l'art poétique soit porteur d'une réelle valeur esthétique.

4 Les quelques pages qui vont suivre se proposent donc d'exposer les concepts clefs qui fondèrent la pensée esthétique traditionnelle de l'Inde, tels qu'ils furent formulés par Bharata, à partir du seul champ d'investigation que fournit l'art théâtral. Les textes ultérieurs relatifs à la musique ou à la peinture se rapportent évidemment à cette théorie esthétique comme étant la seule qui puisse pleinement cautionner la valeur artistique d'une œuvre; mais la réflexion s'en tient souvent à un accord de principe, sur la base d'un système de correspondances qui renvoie implicitement au texte de Bharata. L'une des constantes de la pensée traditionnelle de l'Inde repose sur la réductibilité de la diversité des êtres et des choses à un Tout unique dont ils procèdent. Cette conception originelle, omniprésente dans l'art, est manifeste dans le profond respect qu'inspira toujours le Nātya Śāstra. Le caractère généralisateur de sa théorie de l'art explique en partie la reconduction quasi permanente d'un schéma classique de classifications et de concordances qui caractérisa, des siècles durant, la plupart des observations esthétiques concernant ces disciplines.

\section{La permanence d'un principe originel}

5 A l'instar des philosophes grecs qui ne pouvaient dissocier leurs réflexions esthétiques d'une dialectique de la connaissance ultime du sensible, le Nātya Śāstra poursuit sous un angle particulier l'enseignement contenu dans la pensée originelle des textes sacrés des $V e d a^{6}$. Cette doctrine, qui se développera ultérieurement en systèmes philosophiques et courants religieux, mérite d'être ici brièvement explicitée, car elle est au cœur de toutes les spéculations de la pensée traditionnelle sur l'art. Les textes des Veda, constitués de quatre recueils d'hymnes rituels et sacrificiels, sont les dépositaires de la somme des connaissances sacrées qui fut révélée aux hommes. Cette vérité éternelle est exprimée dans une langue qui est elle-même divine, la langue "parfaite" (c'est le sens du mot samskrita) puisqu'elle fait partie intégrante de la Révélation. L'homme fut alors en possession d'un corps d'enseignements qui lui permettait de résoudre concrètement une préoccupation existentielle permanente, celle de son devenir. Le contenu des Veda 
dévoile à l'homme la possibilité d'atteindre cette attitude inaugurale en se situant par rapport à un principe absolu, conscience omniprésente et illimitée de l'univers, le Brahman, dont il est le reflet microcosmique et vers lequel il doit tendre à s'identifier. Le Brahman est regardé comme cette intelligence cosmique qui exprime l'ordre universel dans le monde manifesté (Renou 1978:132).

Concomitant au principe du Brahman, existe dans l'homme un principe immortel et corrélatif, l'ātman (littéralement soi-même) désignant le Soi, image réflechie du Brahman. La voie expérimentale et transformatrice des préceptes édictés dans les stances des hymnes et enseignée au travers de la doctrine brahmanique, vise d'abord une éducation intégrale de l'homme qui l'aidera à se réintégrer au Tout dont il est issu (Biardeau 1981:86). Les textes védiques enseignent aux hommes un système structuré de valeurs hiérarchisées qui régit l'ensemble de la société à un niveau individuel et collectif. Ces valeurs, exprimées sous forme d'étapes qui jalonnent la vie de l'individu, sont de la plus haute importance pour tous, puisqu'elles sont les garantes de toutes les espérances du devenir existentiel. Elles sont regroupées en quatre sortes de mobiles de la conduite humaine: artha, les biens et les choses matérielles; kāma, les désirs et les passions; dharma, l'ordre socio-cosmique, dynamique de la loi universelle qui régit le monde manifesté mais aussi le devoir à un niveau individuel et mokșa, le but ultime de tout être humain, la libération des trois stades précédents.

7 Il se dégage des enseignements des Veda une idée qui sera omniprésente et commune à toutes les spéculations ultérieures, celle qui insiste sur la nécessité d'une transformation essentielle de soi-même pour une réelle et profonde communion avec un ordre cosmicisé du monde phénoménal. En d'autres termes, la doctrine brahmanique qui s'en tient à la Révélation et met l'accent sur une participation active au rituel, veut conduire les potentialités dynamiques de l'homme à trouver leur aboutissement dans le dévoilement de la vrai nature de son Soi, l'ātman. Au travers de ces enseignements, le véritable et ultime mobile de la conduite humaine, mokșa, consiste donc à recouvrer la pureté de son ātman pour s'unir, s'identifier au Brahman, et par là même se dégager du cycle des renaissances et atteindre la délivrance (ibid:45-76).

\section{Le but de l'art}

8 La réflexion esthétique témoigne d'une préoccupation ontologique identique à celle des enseignements sacrés et marque sans conteste son appartenance profonde à la pensée originelle de l'Inde. Dès les premiers vers d'introduction du Nātya Śāstra, l'art est défini comme étant non seulement source de plaisir et moyen de connaissance, mais aussi comme étant la seule activité instauratrice destinée à tous les hommes, sans distinctions de castes, alors que le savoir consigné dans les textes révélés était jusque là réservé aux seules castes supérieures.

"Revêtu des manifestations diverses de la vie, incarnant les phases diverses de l'action, j'ai fait ce Théâtre conforme au mouvement du monde.

Pour les hommes supérieurs, inférieurs et moyens, il est un réceptacle de toute activité; il engendre des enseignements utiles, et, de l'énergie tendue au relâchement du jeu, il donne toutes les joies.

Ainsi, par les Saveurs, Sentiments et tous les modes de mouvement, ce Théâtre sera pour tous une source d'enseignements.

Au Savoir sacré, à la science et aux mythes, il fournira un lieu d'audience, et à la foule un divertissement: tel sera ce Théâtre ${ }^{8}{ }^{8}$ 
Conforme au mouvement du monde, l'art dramatique est conçu comme un cinquième $V e d a^{9}$ et rappelle à l'homme oublieux des fondements de la doctrine l'existence des lois universelles qui gouvernent toute la manifestation mondaine. L'art devient alors un nouveau moyen de connaissance permettant d'appréhender la vérité sans pour autant se départir de son rôle de divertissement. L'expérience esthétique décrite dans le Nātya Śāstra amène le spectateur sensible, celui qui perçoit la représentation artistique détaché de toute implication personnelle, à reconnaître la profonde nature des lois universelles contenues dans les quatre mobiles de la conduite humaine. Mokșa étant bien entendu la seule vraie finalité qui sera jamais assignée à l'art. Mais si l'art est un moyen d'accès à la connaissance, sa fonction de divertissement et de plaisir est essentielle car c'est elle qui y conduit. Dans la pensée originelle du brahmanisme, les notions de connaissance et de délectation ne sont en effet guère différentes l'une de l'autre, pour ne pas dire confondues (ibid:110). Leur conjugaison dans le domaine de l'art atteste de l'aboutissement logique d'un système de pensée qui trouve ici son expression la plus épanouie.

10 Ainsi, la plénitude de l'expérience esthétique est-elle regardée comme un état particulier de la conscience qui se dévoile à elle-même dans la délectation de l'œuvre et qui par conséquent, accède à un ordre supra-ordinaire de la réalité du monde sensible. La substance de l'œuvre, donnée à être goûtée comme une saveur, est perçue selon un mode d'appréhension direct et immédiat assurant à l'expérience une authentique valeur de connaissance. Cette expérience sera même par la suite rapprochée de l'expérience extatique du mystique ${ }^{10}$ qui, dans sa complète absorption, est plongé dans un moment de ravissement où il goûte à la substance de l'Absolu, le Brahman.

\section{Le rasa, essence de l'art}

11 La tradition esthétique de Inde n'a jamais valorisé la notion de Beau au point de l'ériger en théories et d'en faire, comme en Occident jusqu'à la fin du XIX siècle, un maître mot dont la seule valeur puisse déterminer et fonder tout jugement esthétique. Peu considéré dans l'exposition classique, le Beau ne caractérise que l'appréciation formelle d'une œuvre selon des critères de mesure en conformité avec un ensemble de lois qui détermine la nature des êtres et des choses ainsi que leurs formes ${ }^{11}$. La beauté formelle est le plus souvent considérée comme un ornement (alạ̣kāra), un accessoire uniquement destiné à rehausser l'œuvre, dont il ne faut pas abuser et qu'il convient de traduire à l'aide des canons traditionnels propres à chaque discipline artistique. Comme pour les néoplatoniciens d'Alexandrie, le Beau est avant tout la puissance émouvante du Vrai. Il ne saurait en aucun cas être une qualité discernable objectivement et demeure dans son acceptation platonicienne totalement indépendant des réactions et passions de l'esprit. Les théoriciens hindous associèrent et subordonnèrent l'idée du Beau à la seule expérience esthétique qui puisse intimement révéler le contenu de l'œuvre d'art, l'expérience délectable du rasa. Le mot rasa dont la racine ras veut dire goûter, signifie donc ce qui est goûté, savouré et le terme est généralement traduit par le mot « saveur ».

12 L'idée première et fondamentale du rasa, qui sous-tend toute la pensée esthétique de l'Inde, est une notion récurrente au thème central des Veda. Par la voie empirique du culte et du sacrifice, les hymnes sacrés proposent, à l'homme célébrant le rituel, de communier intimement avec le divin en ingérant, par voie de retour, l'offrande 
consacrée. Cette expérimentation, à la fois synonyme d'assimilation et de transformation, sera reformulée avec les premières Upanișad ${ }^{12}$ qui exhorteront l'homme à s'engager dans une expérience davantage pragmatique le conduisant à la découverte de lui-même, et par conséquent à la compréhension de l'ordre du monde. La nature de l'expérience esthétique du rasa telle qu'elle est décrite dans la tradition médiévale, relève d'un procédé et d'une finalité identiques: le spectateur ou l'auditeur sensible qui participe activement à l'œuvre en l'intériorisant se détache des émotions ordinaires qui gouvernent sa vie quotidienne, absorbe la saveur de cette œuvre et, en même temps qu'il la goûte, s'expérimente luimême dans le ravissement d'un état mental supra-ordinaire (Masson \& Patwardhan 1970 vol. 1:27-28). «On appelle Saveur la perception immédiate, par le dedans, d'un moment ou d'un état particulier de l'existence, provoquée par la mise en œuvre de moyens d'expression artistique. Elle n'est ni objet, ni sentiment, ni concept; elle est une évidence immédiate, une gustation de la vie même, une pure joie de goûter à sa propre substance, tout en communiant avec l'autre, l'acteur ou le poète » (Daumal 1970:15).

Le rasa ne peut avoir d'existence antérieure à sa perception; il est totalement indépendant de toute activité intellectuelle et correspond à un moment de conscience et de communion unique qui en font une expérience sui generis. Seul celui qui manifeste une grande sensibilité et qui est capable d'une réponse par sympathie, le sahrdaya ${ }^{13}$, peut ressentir la profonde finalité de cette expérience et découvrir dans la gustation du rasa une joie intense (ānanda), dont la nature transcendentale, souvent mise en correspondance avec l'expérience mystique, se situe au delà des émotions agréables de la vie courante. «La jouissance esthétique est donc encore un cas de dépossession de soi, où le moi s'oublie dans la contemplation des émotions et des sentiments représentés sur la scène ou dans le poème" (Biardeau 1981:141). Bharata insiste sur la nature supraordinaire (alaukika) de cette expérience esthétique. Les consignes théâtrales exposées dans le Nātya Śāstra précisent que les émotions et les sentiments mis en scène sont analogues à ceux de la vie de tous les jours, mais qu'ils sont détachés du contexte psychoaffectif dans lequel ils sont plongés quotidiennement. Investie d'une dimension « extraordinaire ", la représentation artistique de ces sentiments est destinée à éveiller et entretenir chez le sahrdaya un état mental, libéré des préoccupations mondaines, qui le prépare à la délectation du rasa.

\section{La théorie du rasa}

C'est dans le sixième chapitre du Nātya Śāstra, le Rasādhyāya, que Bharata énonce sa théorie du rasa, qui sera par la suite maintes fois glosée, alimentant ainsi de nombreuses polémiques au terme desquelles l'authenticité de l'idée originelle ira même jusqu'à se perdre dans un dédale de rhétorique (Krishnamoorty 1979:253). Il faudra attendre le X $\mathrm{X}^{\mathrm{e}}$ siècle pour que cette théorie esthétique retrouve dans un premier temps un écho particulier en poésie avec Ānandavardhana et son concept de suggestivité, dhvani; mais ce seront surtout les pénétrantes réflexions d'Abhinavagupta qui redéfiniront pleinement, à partir des éléments contenus dans le Rasādhyāya, les idées promulgant définitivement la suprématie de l'expérience du rasa pour en faire une véritable philosophie de l'art. Les théoriciens et commentateurs postérieurs à Abhinavagupta se contenteront tous de reprendre l'idée maîtresse de son œuvre, n'ajoutant rien de vraiment essentiel à cette théorie du rasa. 

participent au processus d'élaboration et d'avènement du rasa, ainsi que les diverses règles qui en découlent et que doivent respecter les acteurs, afin qu'il puisse être perçu par le spectateur. Bharata énumère huit saveurs distinctes ${ }^{14}$ qui, dans la complémentarité de leurs étendues respectives, recouvrent tout le spectre psychologique de l'activité humaine. D'après les sentiments manifestés dont ils sont les notions intuitives, ces huit rasa sont les suivants: l'érotique (śringāra), le comique (hāsya), le pathétique (karuna), le furieux (raudra), l'héroïque (vīra), le terrifiant (bhayānaka), le répugnant (bïhasta), et le merveilleux (adbhuta). Bharata en explique le mode d'apparition en usant d'une analogie culinaire:

« De même que les gourmets prennent plaisir à déguster la saveur d'une nourriture préparée à partir de plusieurs épices, le spectateur sensible savoure les émotions dominantes suggérées par l'action des diverses manifestations représentées... $\gg^{15}$.

L'expérience esthétique n'est donc possible que par l'action des diverses manifestations représentées, c'est-à-dire par un ensemble d'éléments déterminants qui conduisent à la perception du rasa et que l'on appelle bhāva. Reprenant sa métaphore culinaire, Bharata distingue le rôle de ces bhāva:

«De même qu'une saveur épicée est créée à partir de différentes substances, les bhāva, par leurs nombreuses façon d'intervenir, créent les rasa $»{ }^{16}$

Il en précise ensuite la nature dans le chapitre VII qui leur est consacré, le bhāvādyāya:

" On appelle bhāva tout ce qui, grâce aux effets involontaires (transpiration, larmes, voix tremblotante, etc.), expressions du visage, discours et mouvements corporels, suggère ou manifeste les sentiments internes du poète $»^{17}$.

18 Les bhāva constituent donc tout un ensemble de circonstances, de causes et d'effets permanents ou transitoires, dont la représentation dans l'œuvre va susciter chez le sahrdaya l'apparition et la stabilité d'un élément émotionnel essentiel appelé sthāyibhāva. Ce sentiment dominant est le stade ultime du processus évolutif de l'expérience esthétique qui précède et prépare à la gustation du rasa. Les diverses manifestations des bhāva et leurs représentations qui conduisent à la perception du sthāyibhāva, existent dans une relation complexe, principalement basée sur des critères psychophysiologiques. Bharata regroupe l'ensemble de ces bhāva au sein d'une liste qu'il précise être non exhaustive et mentionne également les huit sthāyibhāva qui sont mis en correspondance avec les huit rasa ${ }^{18}$ :

\begin{tabular}{|l|l|l|l|l|}
\hline \multicolumn{2}{|l|}{ Les huit rasa ou saveurs. } & & \multicolumn{2}{|l|}{ Les huit sthāyibhāva ou sentiments dominants } \\
\hline śrnigārā & érotique & $\leftrightarrow$ & rati & amour \\
\hline hāsya & comique & $\leftrightarrow$ & hāsa & rire \\
\hline karuṇā & pathétique & $\leftrightarrow$ & śoka & tristesse \\
\hline raudra & furieux & $\leftrightarrow$ & krodha & colère \\
\hline vīra & héroïque & $\leftrightarrow$ & utsāha & énergie dynamique \\
\hline bhayānaka & terrifiant & $\leftrightarrow$ & bhaya & peur \\
\hline
\end{tabular}




\begin{tabular}{|l|l|l|l|l|}
\hline bibhatsa & répugnant & $\leftrightarrow$ & jugupsā & dégoût \\
\hline adbhuta & merveilleux & $\leftrightarrow$ & vismaya & étonnement \\
\hline
\end{tabular}

19 Les bhāva qui conditionnent l'apparition du sthāyibhāva sont répertoriés en trois catégories pouvant agir réciproquement l'une envers l'autre afin d'aider à instaurer la permanence de ce sentiment. Dans le cadre d'une représentation théâtrale, les vibhāva sont les causes déterminantes de toute situation, se rapportent aux personnages et sont en nombre illimité. Les anubhāva sont également associés aux personnages et constituent les effets. Il faut ranger parmi ces effets les indices physiques à l'intérieur desquels peuvent être représentés certains états émotionnels involontaires comme les tremblements ou la sueur. Ce sont les anubhāva qui conduisent le spectateur à la reconnaissance du sentiment dominant, et leur nombre est également illimité. Viennent enfin les vyabhicāribhāva, les états passagers ou manifestations subsidiaires, dont le rôle est d'aider le sthāyibhāva à s'intensifier, pour faire naître chez le spectateur la perception du rasa. Subordonnés au sthāyibhāva, les vyabhicāribhāva en sont les accompagnements transitoires et ne sont pas inhérents aux personnages. Rasa et bhāva sont liés dans une relation de mutuelle dépendance et Bharata insiste sur le rôle de ces trois catégories de bhāva dont il considère l'action comme la condition sine qua non du processus de gustation esthétique. Soucieux de définir clairement la nature du rapport que les bhāva entretiennent avec le rasa, il utilise une fois de plus un procédé analogique qui lui est cher et déclare:

«De même qu'un roi, accompagné du cortège de nombreux hommes, aussi éminents qu'ils puissent être, reçoit seul le titre de roi, l'émotion permanente seule, accompagnée d'un cortège de vyabhicāribhāva, anubhāva et vibhāva, acquiert le nom de rasa $» .^{19}$

Pour conclure son rasādhyāya et illustrer concrètement son propos, Bharata reprend chaque rasa individuellement et, à titre d'exemple, indique pour chacun d'entre eux un certain nombre de vibhāva, anubhāva et vyabhicāribhāva, qui doivent être représentés d'une manière ou d'une autre pour concourir à l'apparition et à la permanence du sthāyibhāva.

\section{Une renaissance esthétique}

21 Après que Bharata eut donné et précisé tous les éléments qui entrent en jeu pour manifester la présence du rasa, une importante tradition en poursuivit la voie: tradition essentiellement littéraire, au sein de laquelle de nombreux auteurs, à grands renforts de classification et de théorisation, réinterprétèrent, d'une manière souvent fort personnelle, les termes employés et définis par Bharata, au point que leurs contributions semblent avoir plus constitué un obstacle qu'une aide pour éclairer la compréhension de l'expérience esthétique (Krishnamoorty 1979:149). Utilisé en grammaire, le rasa devint presque un terme technique et le double sens qu'il acquit en littérature ajouta une certaine ambiguïté aux diverses interprétations qu'en donnaient déjà la tradition et le langage courant (Masson \& Patwardhan 1970 vol. 1:42). D'autres controverses concernant le rôle et la fonction des bhāva par rapport au rasa ainsi que la localisation de ce dernier chez l'auteur, l'acteur, le personnage ou le spectateur, soulevèrent de nouvelles difficultés qu'il fallait résoudre. L'ensemble de ces problèmes entretint la cohorte déjà nombreuse de 
spéculations et d'abstractions théoriques qui tentaient de codifier un principe qui, de par sa nature intuitive, ne pourrait jamais l'être. Comme Bharata l'indiquait déjà:

«Les grands poètes sont ceux qui sont le plus proche du réel. La tendance qui les pousse à se railler des conventions et des règles restrictives est sûrement l'indice de grands artistes $»^{20}$.

Tous les commentaires du Nātya Śāstra qui furent écrits dans la période comprise entre la fin de sa rédaction et le $\mathrm{X}^{\mathrm{e}}$ siècle ont été perdus depuis longtemps. La seule trace existante de ces gloses, le nom de leurs auteurs respectifs et un bref rappel de leurs réflexions sont mentionnés et discutés par Abhinavagupta dans son propre commentaire du Nāṭa Śāstra, l'Abhinavabhāratī.

C'est au Cachemire que naquit au IX siècle un puissant mouvement culturel qui s'éleva contre le dogmatisme et la trivialisation qui entachaient la réflexion esthétique. Le poète Ānandavardhana fut le premier à fustiger l'attitude normative et formaliste de ses prédécesseurs et à réhabiliter la valeur fondamentale du rasa, à ses yeux la seule véritable finalité de l'expérience artistique (Krishnamoorty 1979:63, 166). Il est l'auteur d'un remarquable ouvrage, le Dhvanyāloka, dans lequel sont présentés et expliqués exhaustivement les futurs canons de l'art poétique ${ }^{21}$. Ānandavardhana y développe sa théorie du dhvanii2 (suggestion, résonance) qu'il envisage comme un principe destiné à faire valoir la beauté inhérente à chaque élément du poème, dans le seul but de servir l'expérience du rasa. Ce principe de suggestivitée ${ }^{33}$ est élaboré en une brillante théorie que défendra et expliquera minutieusement Abhinavagupta un siècle plus tard dans son commentaire du Dhvanyāloka, le Locana.

Fig. 1: Rāgīnī Megh Malhār. Ecole du Deccan, vers 1750. Indian Museum, Calcutta.

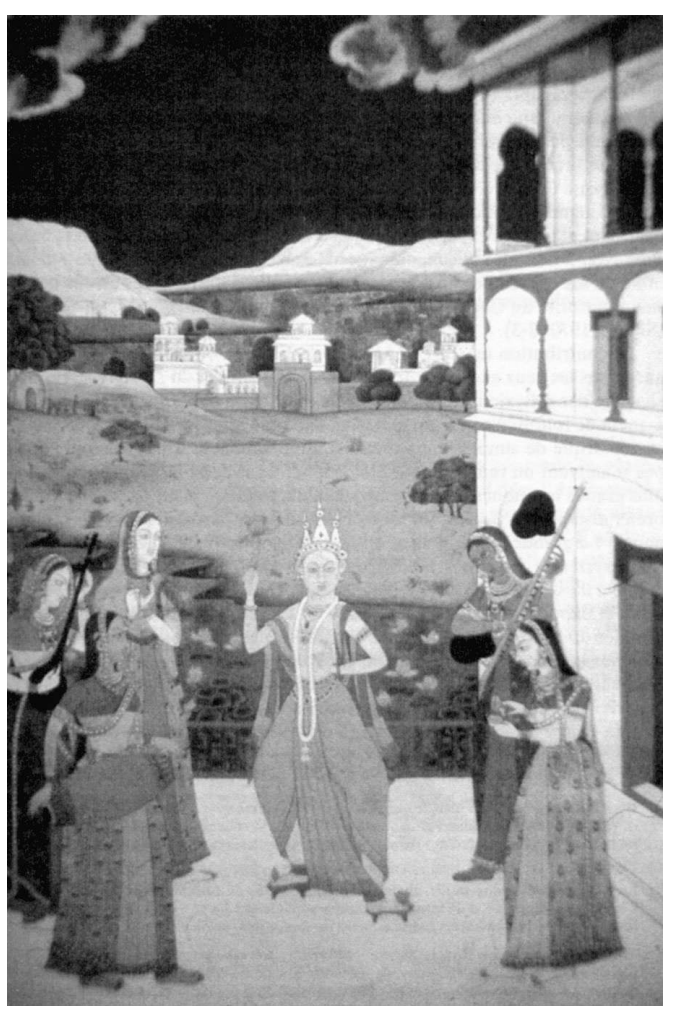

Photo: Philippe Bruguière 
. Son œuvre immense, littéraire, philosophique et métaphysique révèle une pensée hors du commun qui sut pénétrer avec une singulière acuité la nature intime des choses. «Par la profondeur et la subtilité de son esprit, il s'apparente à Maître Eckhart, Ruysbroeck, Saint Jean de la Croix. Simple et direct comme eux, il jouit en plus d'une totale liberté; il peut exprimer ses certitudes et même ses expériences jusqu'à la limite des possibilités de la parole sans avoir à redouter la condamnation d'une église. Il attira l'admiration unanime de ses contemporains, forçant même celle des ritualistes étroits qui faisaient autorité au Cachemire et que visent maintes allusions de ses œuvres »(Silburn 1970: 2-3).

consignée dans les deux exégèses qu'il fit du Nātya Śāstra et du Dhvanyāloka. Dans chacune des réflexions qu'il porte sur le théâtre ou la poésie, il a le souci permanent de fournir à son argumentation une base philosophique rigoureuse qui se démarque de simples constructions intellectuelles. Abhinavagupta ne fut pas seulement un remarquable philosophe, il fut aussi et avant tout l'un des plus grands mystiques de son temps comme en témoignent les récits de nombreux disciples et fidèles qui, des siècles durant, perpétuèrent ses enseignements. La grande connaissance qu'il avait acquise du shivaïsme et des différents systèmes issus des tantra ${ }^{24}$ explique probablement l'insistance avec laquelle il associe étroitement, dans ses spéculations sur l'art, expérience mystique et expérience esthétique. C'est l'un de ses aînés, Bhatțanāyaka, qui le premier établit un parallèle entre l'extase du yogi et l'expérience du rasa ${ }^{25}$. Abhinavagupta partage pleinement cette opinion qu'il nuance cependant subtilement, discernant les similitudes et les différences qui caractérisent ces deux expériences (Gnoli 1968:xxxix-xl). Il réinstaure également l'idée fondamentale du Nātya Śāstra, selon laquelle l'art est l'intermédiaire privilégié entre l'homme et le principe absolu qu'est le Brahman et, tout au long de ses réflexions, revient avec insistance sur le caractère supra-ordinaire de l'expérience esthétique et de l'art en général.

Par ses brillants commentaires et ses nouvelles perspectives qui visaient avant tout à élargir le champ d'introspection de l'expérience du rasa, la portée et l'empreinte de la pensée esthétique d'Abhinavagupta furent immenses. Il fut non seulement considéré comme le chef de file de ce vaste courant réformateur qui rétablit définitivement les bases théoriques énoncées par Bharata, mais il fut aussi le premier à proposer une véritable philosophie de l'art en proclamant l'existence d'un neuvième rasa, celui de la sérénité, le śānta rasa ${ }^{26}$. Bien que l'existence de ce neuvième rasa ait déjà été attestée dans un ouvrage jaïn du $\mathrm{V}^{\mathrm{e}}$ siècle ${ }^{27}$ et discutée par certains poètes et dramaturges bouddhistes, sa reconnaissance, longtemps objet de vives controverses, ne fut réellement admise qu'avec la théorie qu'en donna Abhinavagupta ${ }^{28}$.

\section{Le processus de gustation du rasa}

L'une des préoccupations essentielles d'Abhinavagupta concerne le procédé d'élaboration qui conduit au rasa et qu'il analyse en détail dans l'Abhinavabhārati, reprenant point par point chaque commentaire écrit par ses prédécesseurs sur les chapitres du rasādhyāya et du bhāvāadhyāya du Nātya Śāstra. Il en donne une interprétation extensive, récuse ou admet certains points de vue en une brillante démonstration, ajoute ses propres remarques et expose enfin sa théorie du rasa: lorsque le spectateur ou l'auditeur sensible, 
le sahrdaya, assiste à une représentation théâtrale ou lit un poème, sa perception du temps et de l'espace s'en trouve fortement altérée. Toutes considérations ordinaires pour le moment présent cessent. L'œuvre étant le seul objet de son attention, le regard qu'il y porte est d'une tout autre nature que celui qu'il peut exercer en temps normal. Ne se sentant plus directement ou personnellement concerné, l'habituel mélange de désirs et d'anxiétés qui nourrit son égoïsme se dissout et laisse place à une réponse du cœur. Peu à peu, cette réponse s'intensifie et le sahrdaya s'identifie par empathie avec la situation décrite. Son «moi » normal est alors suspendu pour la durée de cette expérience et il réalise soudainement, dans un état sans précédent de calme mental et émotionnel, qu'il est en train de vivre quelque chose qui n'a rien de commun avec les émotions ordinaires qu'il expérimente quotidiennement. La pureté et l'intensité de cet état le transportent dans l'émerveillement du plus grand ravissement (camatkāra) et il goûte à une absolue félicité (rasāsvāda) car il est entré en contact direct avec les replis les plus cachés de son inconscient, là où la mémoire d'une unité primitive entre l'homme et l'univers est toujours puissante. Sans intention, dit Abhinavagupta, le sahrdaya est parvenu au même lieu intérieur que celui qu'occupe le mystique, bien que son but ait été très différent du sien (Masson \& Patwardhan 1969: vii-viii).

La notion de mémoire ancestrale n'est pas une idée propre à Abhinavagupta, elle existait déjà bien avant lui pour avoir été élaborée avec les premiers systèmes philosophiques et discutée par Patañjali dans son célèbre Yogasūtra. Appelée vāsanā, elle correspond à un ensemble de traces résiduelles des existences antérieures qui compose la conformation interne de chaque individu et que celui-ci possède dès la naissance. Ces vāsanā ou impressions latentes d'innombrables émotions expérimentées par le passé au fur et à mesure des renaissances, ont pour Abhinavagupta une importance capitale dans le processus de gustation esthétique. Ce sont elles qui, une fois éveillées ou stimulées par le jeu de l'imagination, vont devenir la conscience intuitive du sahrdaya. Ce sont elles, dit-il, qui distinguent le véritable sahrdaya, celui qui a un cœur, de ceux qui ne peuvent exploiter cette faculté de représentation et qui « au théâtre sont comme les poutres, les murs et les pierres" (Ibid. 1970 vol. 2: 34). C'est en partie grâce à cette mémoire référentielle que le sahrdaya va pouvoir intensifier sa perception du sentiment dominant qui émane de l'œuvre. Le rôle et la combinaison des bhāva (causes, effets et manifestations transitoires) vont donc stimuler la conscience du sahrdaya, réveillant ces $v \bar{a} s a n \bar{a}$ enfouies dans l'inconscient. Celles-ci viennent alors aider à renforcer l'impression du sentiment dominant et participent ainsi à sa perception. 
Fig. 2: Détail d'une peinture comprenant les trente-six rāga principaux. Jaipur, milieu du XIX ${ }^{\mathrm{e}}$ siècle. Collection Kumar Sangra Singh.

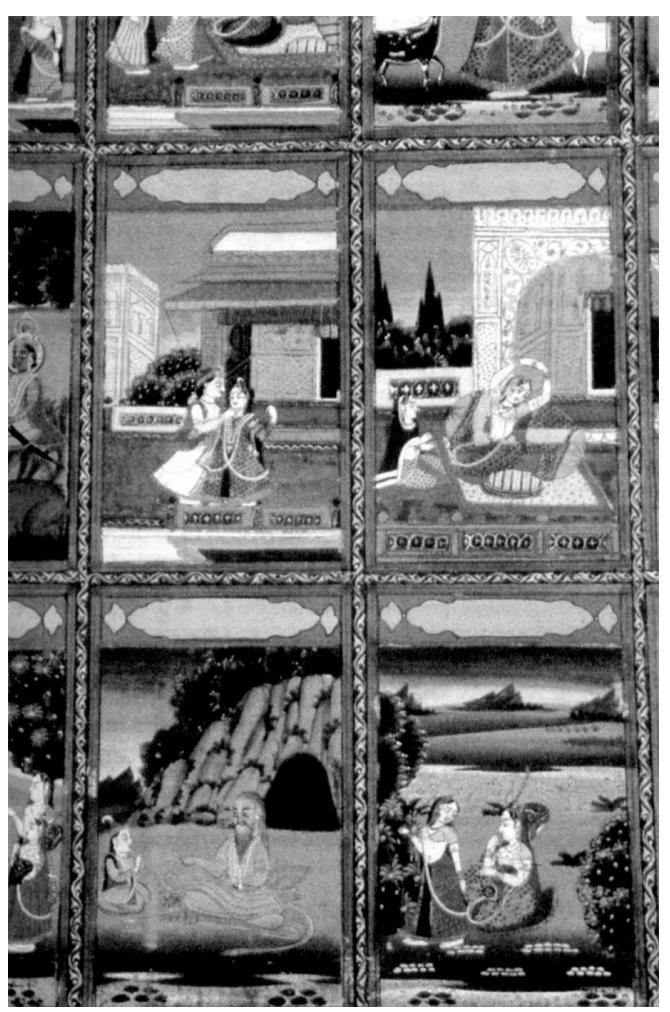

Photo: Philippe Bruguière

Dans le cours de son analyse du processus esthétique, Abhinavagupta explique comment la présence de ce sentiment dominant est ensuite activée par une réponse du cœur ( hrdayasamvāda) ou réponse par empathie ${ }^{29}$. Cette étape décisive est celle qui, avec l'aide des vāsanā, transporte le sahrdaya dans un élan dynamique d'universalisation ( sādhāraṇikaraṇa) jusqu'à un niveau supérieur, celui d'une totale immersion dans l'œuvre, état qui préfigure l'imminence de l'expérience du rasa et qu'Abhinavagupta nomme l'identification (tanmayibhavana). S'adressant aux poètes et à leur lecteurs, il précise une fois de plus les qualités internes du sahrdaya en déclarant:

«Parce que le miroir de leur cœur a été poli sans cesse grâce à une pratique constante de la poésie, ceux qui sont capables de s'identifier avec le contenu du poème et répondent par affinité du fond de leur cœur, (ceux-là) sont reconnus comme sahrdaya $" .^{30}$

En référence directe à cette stance, il cite ensuite ce vers notoire du Nātya Śāstra:

"L'extériorisation (bhāva) d'une émotion qui va directement au cœur, est la source du rasa. Le corps s'embrase alors comme le bois sec au contact du feu $» .^{31}$

Lorsqu'il discute de ce principe d'identification dans un contexte philosophique, il l'assimile au plus haut niveau de réalisation de l'être, celui qui coïncide avec la prise de conscience de son propre soi, ātman. Aucun rejaillissement ou aucune nouvelle étape ne peuvent succéder à cet état où tout se résorbe dans la vacuité de la conscience émerveillée. En revanche, la nature de cette identification est différente dans le domaine esthétique car elle est immédiatement suivie de l'ultime stade du processus: la délectation du rasa (Gnoli 1968:61-62). Abhinavagupta fait remarquer qu'en réalité, le sahrdaya ne s'identifie pas totalement à l'œuvre mais qu'il ménage une sorte de distance qui, dans son 
rapport à l'objet esthétique, est ce que l'on nomme l'expérience du rasa. C'est à l'intérieur de cette distance que s'inscrit le ravissement émerveillé (camatkāra), terme qu'il utilise fréquemment dans ses écrits philosophique et qui correspond ici à ce moment intense où le flot des $v \bar{a} s a n \bar{a}$, mêlé au pouvoir de l'imagination ${ }^{32}$ (pratibhā), déclenche avec le contenu émotionel que suscite l'œuvre, une expérience de pure délectation.

\section{Rasa et rāga}

Elevée au rang d'une philosophie de l'art par Abhinavagupta, la théorie du rasa se développa en une dialectique réalisant une parfaite adéquation entre les considérations techniques dont procèdent le mode opératoire de l'expérience esthétique et la nature intrinsèque d'un état de l'être transporté dans la délectation de l'œuvre. Mais qu'en est-il donc de cette théorie généraliste, centrée autour de l'art dramatique et de la poésie, lorsqu'il s'agit de l'envisager dans le domaine musical? Est-elle réellement applicable selon des moyens et un processus de développement identiques? Faut-il considérer, comme Bharata semble l'indiquer, que seule la finalité du rasa importe et que, malgré leur singularité, les moyens spécifiques mis en œuvre dans telle ou telle discipline sont tous assujettis à un système de correspondances qui se rapporte à la théorie initiale? Si cette dernière, conçue autour d'un art descriptif, peut être concevable pour toute autre forme artistique qui fait appel à une même capacité d'objectivation face à un objet conceptuel, il en va tout autrement pour la musique, dont le caractère éphémère appartient essentiellement au domaine de l'évocation. En d'autres termes, un poème empreint d'allégresse, une couleur bleue ou un personnage exprimant la colère seront ressentis comme tels ou du moins signifieront-ils concrètement ce qu'ils sont; mais peut-on affirmer qu'un mouvement mélodique exprimant la tristesse sera nécessairement perçu comme tel? D'autres difficultés d'ordre historique et socio-culturel apparaissent également dans cette problématique. Lorsque par exemple Bharata indique à la fin d'un chapitre consacré à la musique ${ }^{33}$ les structures musicales corrélatives aux rasa, il fait état d'une pratique musicale qui était déjà devenue obsolète lorsque, dans le courant du premier millénaire, furent composés les deux seuls ouvrages musicaux ${ }^{34}$ qui soient parvenus jusqu'à nos jours et qui en donnent un commentaire.

Ces écrits, comme l'ensemble de la littérature musicale en sanskrit qui s'épanouira à partir du XI ${ }^{e}$ siècle, apportent leurs diverses contributions en s'appuyant sur le contenu musicologique du Nātya Śāstra dont ils s'inspirent également pour reproduire un système identique d'homologations et de correspondances. Ainsi, les sept notes qui constituent l'échelle musicale sont respectivement associées à une divinité tutélaire, un sage, un centre vital du corps humain, un rasa, une couleur, une saison, une planète, etc. La composition de ces textes suit en général un agencement prédéfini: vient d'abord une déclaration liminaire glorifiant l'un des dieux de la triade du panthéon hindou, un hommage est ensuite rendu à Bharata, et en introduction sont rappelés l'aspect métaphysique du son ainsi que les fondements de la pensée brahmanique. L'art musical, à l'instar du théâtre originel, présenté comme le véhicule privilégié pour atteindre les plus hautes réalisations spirituelles, est alors discuté en détail en différentes sections. La précision de l'argumentation et de la terminologie, les analyses circonstanciées de chaque composant et les interminables listes de classifications sont étonnantes à plus d'un titre. L'élément dynamique autour duquel gravite la théorie musicale est évidement le rāga, entité mélodique complexe qui ne saurait être assimilée à une gamme, à une échelle ou à 
un mode mais qui correspond plus exactement à une atmosphère ou un climat musical particulier. Ce mot vient de la racine sanskrite ranga qui signifie couleur, et par extension le răga est « ce qui colore (d'émotion) l'esprit ». Le seul objectif du musicien est donc de rendre ce climat musical suffisamment convaincant afin qu'il puisse « colorer » l'esprit de l'auditeur sensible et susciter en lui une réponse à la couleur émotionnelle évoquée. Cependant, l'attention portée au domaine de l'esthétique dans les ouvrages musicaux est presque toujours réduite à sa plus simple expression, celle d'une traditionnelle concordance entre les notes ou les rāga et le rasa qu'ils expriment ${ }^{35}$. Le schéma relationnel est toujours reconduit de la même façon, mais l'identité des rāga et rasa mis en correspondance diffère parfois sensiblement d'un texte à l'autre, même lorsqu'ils sont chronologiquement très proches.

Ce n'est que dans le courant du XIII ${ }^{\mathrm{e}}$ siècle que les rāga seront classifiés pour la première fois selon un système qui les distingue en genre masculin, féminin et neutre (Gangoly 1948: 22, Nijenhuis 1977: 14). Cette tentative est basée sur le rasa associé à chaque mélodie et préfigure la classification traditionnelle qui sera adoptée du XVI jusqu'au début du XX ${ }^{\mathrm{e}}$ siècle. Le corpus des rāga est alors répertorié en rāga masculins accompagnés de leurs épouses (rāginī) et de leurs enfants (putra). Dès le VIII siècle, des descriptions imagées ( dhyāna) sont établies pour préciser l'identité des rāga ainsi que celle de leur rasa (Gangoly 1948:106). Ces descriptions, composées à l'origine de quelques vers en sanskrit, seront par la suite reformulées sous forme de quatrains ou couplets en langues vernaculaires (appelés dohā en hindi), afin de toucher un plus important public. Leur contenu allégorique est destiné à évoquer, chez le musicien comme chez le sahrdaya, le sentiment dominant du rasa, le sthāyibhāva. Les dhyāna semblent avoir déjà existé dans les textes les plus anciens ${ }^{36}$ mais ils se rapportent alors exclusivement aux divinités ${ }^{37}$ qui président aux rāga. Ces personnages divins seront remplacées plus tard par la représentation de héros ( $n \bar{a} y a k \bar{a}$ ) et héroïnes (nāyikā) empruntés à l'art dramatique et qui incarneront le sentiment dominant du rasa ${ }^{38}$. Bientôt, en raison d'un certain nombre de confusions occasionnées par l'apparition de nouveaux rāga ainsi que par le développement de la théorie et l'évolution des pratiques musicales, la seule description picturale d'un rāga ne sera plus suffisante pour aider à la représentation mentale du sentiment dominant. C'est ainsi qu'outre cette évocation imagée, sera proposée pour chacun d'eux une description mélodique complémentaire qui sera étroitement associée au dhyāna ${ }^{39}$.

Parallèlement à cette littérature musicologique vont apparaître au $\mathrm{XV}^{\mathrm{e}}$ siècle les premiers textes des rāgamālā, recueils composés de dhyāna, qui deviendront très populaires lorsqu'ils regrouperont en leur sein de délicates séries de peintures illustrant les rāga, les célèbres miniatures appelées rāginī. Ces rāgini tout comme les dhyāna, sont inspirées d'une très riche littérature poétique et ne servent manifestement aucun propos musical. Les couleurs employées comme la symbolique représentée sur ces miniatures ne se rapportent en aucun cas aux systèmes de correspondances des ouvrages musicaux. L'unique fonction musicale des rāginī au dos desquelles est en général copié le dhyāna ou dohā du rāga, consiste à fournir une description visuelle du rasa (Ibid:102).

Le postulat corrélatif, qui durant de nombreux siècles maintint en contact l'élément musical avec une divinité, une caste, une couleur, etc., perdit graduellement de son autorité à partir du milieu du XVI ${ }^{\mathrm{e}}$ siècle. Lorsque les caractéristiques mélodiques du rāga évoluèrent encore vers de nouvelles interprétations, conduisant la tradition à se scinder en deux systèmes musicaux distincts, l'un au nord et l'autre au sud de l'Inde, la reconnaissance du rasa par la seule indication d'une correspondance, s'en trouva d'autant 
plus affectée. Dès lors, la forme mélodique du rāga, ses dhyāna ainsi que les rāginī qui les accompagnent, devinrent les seuls éléments tangibles pouvant indiquer le caractère esthétique du rāga. La relation rāga - rasa perdit toute crédibilité dans la tradition carnatique, tandis qu'au nord, le sentiment du rāga et le moment propice du jour ou de la nuit pour son exécution demeurèrent deux constantes scrupuleusement respectées. La théorie classique du rasa semblerait pouvoir trouver ici une interprétation satisfaisante qui ne rencontre guère d'obstacles s'il ne fallait auparavant éclaircir un point qui présente quelques difficultés. Lorsque Bharata met en correspondance structures mélodiques et rasa, il n'identifie pas celles-ci aux rāga pour la simple raison que le sens musical de ce terme rāga n'apparaîtra qu'ultérieurement, entre le $\mathrm{V}^{\mathrm{e}}$ et le $\mathrm{VIII}^{\mathrm{e}}$ siècle avec l'ouvrage de Matanga, le Bṛhaddeśi. D'autre part dans l'énoncé de sa théorie, il ne distingue pas le sthāyibhāva du rasa, comme le fera plus tard Abhinavagupta. Il précise simplement que ce sentiment dominant se transforme en rasa lorsqu'il est goûté par le spectateur. Pour Abhinavagupta, le sahrdaya répond d'abord par empathie au sentiment dominant et s'identifie ensuite à lui grâce à ses propres vāsanā. Ce n'est que lorsqu'il est totalement immergé dans le sthâyibhâva qu'advient l'expérience sui generis du rasa. Ce sthāyibhāva qui appartient au domaine de la pensée discursive est donc distinct du rasa (Gnoli 1968:80). Or, dans les textes musicaux postérieurs au Bṛhaddeśi, l'équivalent des structures musicales (jāti) considérées par Bharata puis les rāga, lorsqu'ils sont définis en tant que tels, sont mis en corrélation avec les rasa. Mais, si le răga correspond avant tout à un climat musical destiné à colorer l'esprit de l'auditeur, c'est bien un état affectif particulier qu'il veut transmettre et cet état représente un sentiment durable qui n'est autre que le sthāyibhāva. Par conséquent, il serait plus exact de concevoir le rāga non pas comme l'expression directe du rasa, mais comme celle du sentiment dominant qui en précède l'expérience ultime.

Dans cette perspective, il est alors tout à fait concevable de regarder les descriptions poétiques et picturales, les miniatures et formes mélodiques des rāga comme autant de déterminants (bhāva) qui aident le musicien et l'auditeur à s'imprégner de la représentation du sentiment dominant qu'incarne le rāga. Tel fut d'ailleurs le cas par le passé et encore de nos jours, en dépit d'une abondante littérature musicale, le rāga est d'abord identifié par sa forme mélodique (calana) et ses phrases caractéristiques (pakad) plutôt que par les éléments sémantiques qui le caractérisent. Ces derniers déterminent pourtant le caractère du rāga et à ce titre, pourraient aussi être légitimement considérés comme ses bhāva. Un examen rigoureux des différents types d'ornements destinés à mettre en valeur tel ou tel aspect du sentiment du rāga, montrerait par exemple qu'ils peuvent être rangés tantôt parmi les causes (vibhāva), tantôt parmi les effets (anubhāva) qui œuvrent à sa manifestation. Quant au texte chanté qui accompagne chaque rāga, son contenu possède la plupart du temps un double sens littéral et figuré qui se rapporte au caractère du rāga. Mais s'il renferme cette fonction de suggestivité (dhvani) qui atteste de la plus haute valeur poétique, il contribuera à fortifier la présence du sthāyibhāva dans l'esprit de l'auditeur.

39 Si la théorie de Bharata n'a trouvé au cours des siècles qu'un écho relatif dans la littérature musicale, l'idée du rasa demeure en revanche pour le musicien son unique préoccupation lorsqu'il exécute un rāga. C'est d'abord un sentiment particulier qu'il s'agit de transmettre à l'auditeur, et toutes considérations techniques ou théoriques sont reléguées au second plan, lorsqu'elles ne sont pas totalement occultées. La dichotomie qui a semble-t-il toujours existé entre théoriciens et musiciens, est manifeste dans le peu 
d'intérêt que ces derniers ont montré pour les règles et recommandations d'usage formulées dans les textes. Des siècles durant, les théoriciens s'efforcèrent de définir avec précision l'intonation des rāga, spécifiant minutieusement la hauteur des altérations microtonales de chaques notes. En dépit de tous ces efforts, les musiciens n'accordèrent jamais aucune attention à de telles instructions pour déterminer l'intonation propre à chaque rāga. Leurs seuls critères de référence étaient ceux que leurs aînés leur avaient transmis oralement au cours d'un long apprentissage et durant lequel ils avaient fait la patiente acquisition d'un savoir-faire sophistiqué. C'est ainsi que la plus grande importance était accordée à la couleur spécifique de chaque rāga, nuance qui leur avait été révélée jusque dans ses moindres détails. Aussi se considéraient-ils légitimement comme les seuls gardiens pouvant assurer la pérennité de l'identité mélodique du rāga et par conséquent de son rasa. En tout cas en était-il encore ainsi au cours des dernières décennies du XIX ${ }^{e}$ siècle. La parfaite expression du rasa était pour le musicien le maître mot de l'interprétation du rāga et peu lui importaient les prescriptions théoriques de l'intonation. Il faut ajouter à cela le caractère relatif de la justesse microtonale qui pouvait être très variable d'une école à l'autre. De récentes études ont d'ailleurs souligné combien ces problèmes d'intonation si chers aux musicologues devaient être reconsidérés ${ }^{40}$. Ces travaux montrent que l'interprétation d'un même rāga par deux musiciens unanimement respectés, peut conduire pour les mêmes notes, à des différences d'intonation de cinquante cents, voir plus. Pourtant dans les deux cas, ni l'identité du rāga, ni le sentiment évoqué dans l'esprit des auditeurs n'ont posé l'ombre d'un doute.

$\mathrm{Au}$ début de notre siècle, un musicologue inspiré ${ }^{41}$ entreprit la gigantesque tâche de collecter auprès des plus grands maîtres un répertoire qui, pour de multiples raisons socio-culturelles, risquait fort de disparaître. Il put ainsi réunir un nombre impressionnant de rāga accompagnés de leurs compositions, qu'il classifia par la suite en un nouveau système toujours d'actualité. Malgré l'intérêt évident d'une telle entreprise, dont il publia les résultats en une série d'ouvrages très documentés et destinés à un enseignement à présent institutionnalisé, l'interprète d'aujourd'hui accorde toujours la plus grande attention au rasa du rāga. S'il s'exprime en anglais, il utilisera plus volontiers le mot mood pour qualifier ce qu'il ressent, ce sentiment qu'il veut transmettre et qu'il identifie au rasa. Il est probable que l'homologation du sentiment au rasa, entretenue dans la littérature et comprise comme telle par les musiciens ait entériné l'acception courante selon laquelle le răga exprime le rasa. Certains artistes vont même au-delà de ce consentement et revendiquent la capacité d'user de tel ou tel rasa selon leur état d'âme, quel que soit le rāga choisi.

Cependant, l'auditeur sensible éprouvera toujours une intime émotion esthétique lorsque, dans la profondeur d'un timbre, l'assouplissement d'une courbe sonore ou la fulgurance d'un trait mélodique, il découvrira pour un instant, dans une joie incomparable, l'indicible présence de l'œuvre. Peu importe la connaissance discursive ou conceptuelle qu'il peut avoir de l'art musical, l'expérience intuitive du rasa n'est en aucune manière assujettie aux canons esthétiques et normes artistiques qui sont supposés y conduire. Une œuvre d'art digne de ce nom porte virtuellement en elle cette présence qui se dévoile, comme l'expliquent Bharata et Abhinavagupta, dans le ravissement d'une expérience supra-ordinaire où le sahrdaya goûte également à sa propre saveur existentielle. Source de plaisir et moyen de connaissance, l'art est proclamé instaurateur d'une vérité ontologique. Vérité qui se révèle dans la seule expérience du rasa: ce fugitif instant de grâce où, dans un murmure de délectation, le cœur chavire dans le divin. 


\section{BIBLIOGRAPHIE}

BIARDEAU Madeleine, 1964, Théorie de la connaissance et philosophie de la parole dans le brahmanisme classique . Paris, La Haye: Mouton.

BIARDEAU Madeleine, 1981, L'Hindouisme. Anthropologie d'une civilisation. Paris: Flammarion.

BRUGUIERE Philippe, 1987, Poétique et esthétique dans l'Inde Ancienne: Observations sur la théorie du rasa. Thèse non publiée. Université de Paris IV-Sorbonne.

COOMARASWAMY Ananda Kantish, 1926, Pour comprendre l'art hindou. Paris: Bossard.

DAUMAL René, 1970, Bharata. Paris: Gallimard.

GANGOLI O. C., 1948, Rāgas \& Rāginīs. Bombay: Nalanda Publications.

GNOLI Raniero, 1968, The Aesthetic Experience According to Abhinavagupta. Varanasi: Chowkhamba Sanskrit Series Office.

GODEL Roger, 1976, Socrate et le sage indien. Paris: Les Belles Lettres.

JAIRAZBHOY Nazir A., 1963, « Intonation in Present-day North Indian Classical Music », Bulletin of the School of Oriental and African Studies. University of London, XXVI, 1: 119-132.

KAUFMANN Walter, 1965, « Rasa, Raga-Mala and Performance Time in North Indian Ragas », Ethnomusicology, IX (3): 272-291.

KAUFMANN Walter, 1968, The Ragas of North India. Bloomington: Indiana University Press.

KRISHNAMOORTY Keralapura, 1979, Studies in Indian Aesthetics and Criticism. Mysore: DVK Murthy. KRISHNAMOORTY Keralapura, 1982, Dhvanyāloka of Ānandavardhana . Delhi: Motilal Banarsidass.

MASSON Jeffrey, L., PATWARDHAN Madhav Vinayak, 1969, Santarasa and Abhinavagupta's Philosophy of Aesthetics. Poona: Bhandarkar Oriental Research Institute.

MASSON Jeffrey, L., PATWARDHAN Madhav Vinayak, 1970, Aesthetic Rapture vol. I and II. Poona: Deccan College.

NAYAR Sobhana, 1989, Bhatkande's Contribution to music. Bombay: Popular Prakashan.

RAGHAVAN Venkatarama, 1975, The Number of Rasa-s. Madras: The Adyar Library \& Research Centre [3rd ed].

RENOU Louis, 1978, L'Inde fondamentale. Paris: Herman.

REVUE D'ESTHETIQUE, 1975, Il y a des poètes partout. Paris: UGE [10/18].

SILBURN Lilian, 1970, Hymnes de Abhinavagupta. Paris: De Boccard. [Publication de l'Institut de Civilisation Indienne, 31].

SOURIAU Etienne, 1969, La correspondance des arts. Paris: Flammarion [Science de l'Homme].

SOURIAU Etienne, 1970, Clefs pour l'esthétique. Paris: Seghers.

TE NIJENHUIS Emmie, 1976, The Rāgas of Somanātha, part I and II. Leiden: E. J. Brill.

TE NIJENHUIS Emmie, 1977, Musicological Literature. Wiesbaden: Otto Harrassowitz. 


\section{NOTES}

1. Le sens étymologique du mot esthétique vient du grec aisthétikos qui signifie: ce qui est relatif à la perception par les sens. C'est le philosophe allemand Baumgarten qui le premier utilisa le mot d'esthétique pour définir la discipline qu'il fonda lorsqu'il publia en 1750 son Aesthetica, ouvrage consacré à l'art, son processus créatif et la sensibilité qu'il met en œuvre.

2. «A moins qu'on n'admette à la manière platonicienne un Beau absolu, objet d'une intuition intellectuelle universelle certaine, le Beau n'est pas un fait objectif positivement discernable et propre à donner consistance et solidité à l'étude qu'on peut en faire » (Souriau 1970:47).

3. Edition complète publiée chez Chowkhamba series, Bénarès 1929.

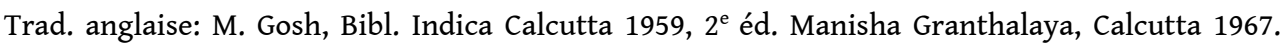
vol. II, Bibl. Indica, The Asiatic Society, Calcutta 1961.

En français: Les chapitres VI etVII, de Paul Regnaud en appendice à La rhétorique sanskrite, Paris 1884 et le Bhāratīya Nātya Śāstram de Joanny Grosset avec préface de P. Regnaud. Annales de l'Université de Lyon, XL 1898. Voir aussi Le théâtre indien de Sylvain Lévi, nelle éd. avec l'article de L. Renou «la recherche sur le théâtre indien depuis 1890 », Bibl. de l'Ecole des Hautes Etudes, fasc. 83, Paris 1963. Pour une introduction à l'art poétique et l'esthétique de l'Inde ainsi qu'une traduction et commentaires du chapitre I du Nāṭa Śāstra, voir René Daumal, Bharata, NRF Gallimard, Paris 1970.

4. L'identité du sage Bharata demeure cependant une énigme puisqu'à la même époque cinq autres Bharata sont mentionnés dans divers textes. Il faut plutôt regarder le nom de Bharata comme une "étiquette mythique et symbolique, résumant sans doute une tradition d'école particulière » (Daumal, op. cit. p. 15). Il semblerait que cet énorme traité qui ne comprend pas moins de cinq mille vers ait été compilé à partir de textes plus anciens et cela, sur une période de plusieurs siècles.

5. Brahmā, distinct du Brahman, le Principe Absolu, représente avec Śiva et Viṣnu, la trilogie du panthéon hindou. Il ne crée pas mais émet, n'enseigne pas mais révèle, il est l'essence de toute existence.

6. Nātya Śāstra I, 18, Daumal 1970:19.

7. Cet Ātman n'est pas sans évoquer le pur principe d'intelligibilité de la Grèce antique, le Logos, implicitement présent en tout homme et par lequel ce dernier est capable d'appréhender la vérité (Godel 1976:57).

8. N. S. I, 110, 111, 112, 119, Daumal 1970:26.

9. «La substance de toutes les sciences, la mise en œuvre de tous les métiers; de tout cela, en y joignant les Mythes, je fais le Cinquième savoir qui s'appellera Théâtre » N. S. I, 15, Ibid:19.

10. Cette idée est identique à celle de Plotin, le chef de file de l'école néo-platonicienne, «qui voit dans l'expérience esthétique des états de l'âme comparables à ceux qu'atteint l'extase mystique avec ses connaissances intuitives d'un genre ineffable » (Souriau 1970:14-15).

11. Ces lois naturelles, explicitées dans le système philosophique du Sāṃkhya, se manifestent en trois qualités primordiales, les guna. La plus grossière, tamas, correspond à un mode de pesanteur, d'inertie et de passivité. C'est une force de limitation, de chute, prépondérante dans le monde inorganique. Rajas représente une force d'expansion, un mode d'action dynamique, de transformation et de mutation. Sous sa prédominance sont formées les énergies vitales, les organes d'action. La troisième, sattva, exprime tout ce qui dévoile la nature stable et lumineuse de l'être. C'est un principe d'intelligence qui se manifeste dans les éléments les plus subtils.

12. Les Upanișad, exégèses des hymnes védiques, furent rédigées au cours des siècles dans un souci de clarification afin de prévenir toute interprétation erronée des textes et pour mettre en garde contre le caractère illusoire d'un savoir purement verbal. 
13. Hṛdaya: le cœur, le siège de la sensibilité esthétique. Le sahrrdaya est le spectateur ou l'auditeur sensible doué d'une capacité d'appréciation et d'immersion dans l'œuvre que ne viennent pas perturber les états mentaux de la vie ordinaire. Il est souvent comparé à l'enfant, chez qui la liberté et la spontanéité des réactions émotionnelles ne sont pas encore troublées par le développement de l'ego (Masson \& Patwardhan 1969:23, 78, 84).

14. N. S. VI, 15, Masson \& Patwardhan 1970 vol. 1:44.

15. N. S. VI, 31, Ibid:46.

16. N. S. VI, 35, Ibid:47.

17. N. S. VII, 2, Ibid:38.

18. N. S. VII, 2, Ibid:44.

19. N. S. VII, Ibid:39.

20. Ibid:42.

21. Cf. Bruguière 1987:151-167.

22. Le concept originel de dhvani est antérieur à Ānandavardhana. Il eut d'abord une importance grammaticale et qualifiait le son concret, la résonance du mot.

23. C'est également par cette idée de suggestivité que Mallarmé définit la poésie comme étant «l'art de peindre non la chose mais l'effet qu'elle produit... le vers ne doit donc pas, là, se composer de mots mais d'intentions et toutes paroles s'effacer devant les sensations " (Revue d'Esthétique 3-4 1975:45).

24. Le terme tantra signifie trame, chaîne et implique l'idée de continuité, de développement. Il s'agissait à son origine, d'un terme générique s'appliquant à un ensemble d'ouvrages très nombreux et variés renfermant les enseignements de source shivaïte ou vishnuite.

25. «On comprend que la jouissance du rasa - rasāsvada - soit mise si facilement en parallèle avec la jouissance du Brahman - Brahmāsvāda -: l'émerveillement - camatkāra - subit que crée l'action dramatique ou le vers est senti comme une expansion de la pensée hors de ses bornes normales, tout comme le yogin ou le mystique - notamment dans le shivaïsme du cachemire accède brusquement à une expérience - camatkāra - où il transcende son ego " (Biardeau 1981:141). Une idée similaire existe dans la pensée néo-platonicienne, lorsque l'âme est saisie d'étonnement en face du beau ou du sacré (Gnoli 1968:xlvi).

26. Śānti, la paix, la quiétude, la sereine tranquillité.

27. L'Anuyogadvara sūtra mentionne neuf saveurs poétiques dont celle de la paix (Raghavan 1975:24).

28. Voir la troisième section de l'Abhinavabhāratī dont la seule traduction disponible est celle qu'en donnent Masson \& Patwardhan (1969:120-163).

29. "L'émotion esthétique naît de l'accord de l'âme avec le mode permanent, donc par empathie et re-création de l'imagination. Elle est essentiellement le produit de l'activité spirituelle du spectateur lui-même [...] et n'est connue que de ceux qui y sont aptes » (Coomaraswamy 1926:83-84). Cette activité d'empathie est à rapprocher de la théorie de l'Einfühlung, chère aux esthéticiens allemands de la fin du siècle dernier. L'Einfühlung ou "imagination pénétrante » consiste à se projeter en pensée dans l'objet contemplé afin d'en percevoir la nature intime, en s'identifiant à lui.

30. Locana, 38, cit. Masson \& Patwardhan 1969:78.

31. N. S. VII, 7, Ibid.

32. Ce pouvoir d'imagination est une autre idée à laquelle Abhinavagupta attache beaucoup d'importance et qui, en matière d'esthétique, fut développée pour la première fois par son propre maître Bhațtatauta. Elle joue un rôle essentiel dans l'interprétation cosmogonique du shivaïsme cachemirien, où elle est identique à la conscience divine en tant qu'émission créatrice. Ici, dans l'art, pratibhā est cette intuition intense, cette vision qui pénètre le cœur des choses, un don divin qui se manifeste chez les plus grands artistes comme chez le sahṛdaya (Bruguière 1987:143-145).

33. N. S. XXIX, 1-13.

Cahiers d'ethnomusicologie, 7 | 1994 
34. Le Dattilam de Dattila-muni et le Brhaddesī de Matanga. Les dates de leur rédaction sont très approximatives. Le Dattilam serait postérieur au Nātya Śāstra mais antérieur au Bṛhaddesī écrit aux environs du VII ${ }^{\mathrm{e}}$ siècle.

35. Le Sangîtaratnākara de Śārngadeva, écrit dans la première moitié du XIII ${ }^{\mathrm{e}}$ siècle, texte phare de la littérature musicale de l'époque médiévale ne fait qu'énumérer les rasa mis en relation avec les notes et les différentes catégories de modèles mélodiques (jāti).

36. D'après les sources tardives qui en font maintes références, le Brhaddesī serait l'un des premiers ouvrages à renfermer de telles descriptions iconographiques. Malheureusement, le texte incomplet ne fournit que de trop rares indications sur cette section manquante.

37. Le support de l'image a de tout temps joué un rôle considérable dans la société traditionnelle. Sa fonction religieuse, à laquelle se rapporte une très riche symbolique, est toute puissante en tant que support d'invocation et de concentration pour communiquer avec le divin.

38. Un traité musical jaïn du début du XIV ${ }^{\mathrm{e}}$ siècle, le Sangitopanisat semble avoir été le premier à décrire des dhyāna de ce type (Nijenhuis 1977:15).

39. Le premier auteur à avoir mentionné ces formes mélodiques fut Somanātha dans son Rāgavibodha qu'il écrivit en 1609. Voir Nijenhuis 1976.

40. Jairazbhoy, Stone 1963:119-132, Kaufmann 1965:272-291.

41. V.N. Bhātkhande (1860-1936), Cf. Nayar: 1989.

\section{RÉSUMÉS}

La pensée esthétique de l'Inde fut énoncée pour la première fois dans le plus ancien traité d'art dramatique, rédigé au début de notre ère, le Nātya Śāstra. Ce texte envisage l'art comme un prolongement des enseignements contenus dans les hymnes des Veda et demeure pour la tradition artistique et littéraire, la première autorité en matière d'esthétique. Attribué au sage Bharata, l'ouvrage traite en détail de toutes les disciplines (danse, poésie, musique, mise en scène, etc.) qui participent à l'art théâtral, révélé par le dieu Brahmā. L'art y est expliqué comme étant à la fois source de plaisir et moyen de connaissance, s'inscrivant ainsi dans la continuité de la doctrine brahmanique. La théorie esthétique de Bharata repose sur la notion de rasa ou saveur qu'exprime une œuvre. Le spectateur sensible en fait une expérience délectable radicalement différente des émotions courantes qu'il peut ressentir dans la vie quotidienne. L'expérience du rasa ne saurait être objectivée mentalement ni même conceptualisée. Elle est une gustation intuitive de l'œuvre et procure une joie ineffable. Par la suite la tradition littéraire enfermera le rasa dans un dogmatisme qui en altérera profondément la nature. Il faudra attendre les $\mathrm{X}^{\mathrm{e}}$ et $\mathrm{XI}^{\mathrm{e}}$ siècles avec l'apparition d'un nouveau courant réformateur et les réflexions de l'un de ses plus brillants représentants, Abhinavagupta, pour retrouver les idées qui élèveront la théorie originelle du rasa au rang d'une véritable philosophie de l'art. L'expérience que procure la gustation du rasa sera même comparée à celle qui transporte le mystique aux cimes de la félicité. Le caractère réductionniste de cette théorie esthétique ne trouvera qu'un faible champ d'application dans le domaine musical où rasa et rāga seront toutefois intimement associés.

The earliest theory of aesthetics in India was formulated in the most ancient treatise of the dramatic arts, the Nātya Śāstra, which dates back to the beginning of our era. This text, which conceives of art as a continuation of the teachings contained in the Vedic hymns, still represents the main source on aesthetics. Attributed to the sage Bharata, the work deals in detail with all 
disciplines (dance, poetry, music, production) involved in drama, as revealed by the god Brahmā. Art is explained as being both a source of pleasure and a means of knowledge, and thus appears as an extension of the Brahmanic doctrine. Bharata's theory of aesthetics focuses on the concept of rasa or flavour given off by a creation. The sensitive spectator thus experiences a delight which differs markedly from the emotions he may go through in ordinary life. Experiencing rasa cannot be objectivised mentally nor conceptualised; it means an intuitive tasting of the work of art and brings everlasting joy. At a later stage, the literary tradition confined rasa to a dogmatic framework which radically altered its nature. Only the emergence of a reform current starting in the tenth century and notably the thought of one of its most brilliant exponents, namely Abhinavagupta, once again fostered ideas which helped carry the original theory of rasa to the level of a genuine philosophy of art. The experience of tasting rasa was even compared to the one which transports the mystic to the heights of religious bliss. The reductionist character of this theory of aesthetics has found a limited field of application in music, in which rasa and rāga are, however, closely related.

\section{AUTEUR}

\section{PHILIPPE BRUGUIÈRE}

Philippe Bruguière reçut une formation musicale avant d'entreprendre en 1975 des études au département de musicologie de l'Université de Paris VIII. C'est en 1980 qu'il se rend en Inde pour étudier l'art de la Rudra vina auprès de Zia Mohiuddin Dagar. Boursier du gouvernement français, il séjourne en Inde jusqu'en 1986 et prépare, conjointement à ses études musicales, une thèse de doctorat sur la tradition esthétique de l'Inde ancienne. Après sa soutenance (Paris IV - 1987), il effectue plusieurs missions de recherche, collectant un important matériel textuel, iconographique et sonore sur la tradition instrumentale de la Rudra vina. Depuis 1990, il est responsable d'un programme de réaménagement de la section musicale du Musée National des Arts Asiatiques - Guimet. Il est chargé de cours en ethnomusicologie à l'Université de ClermontFerrand et, parallèlement à ses activités, donne des récitals de Rudra vina en France et à l'étranger. 